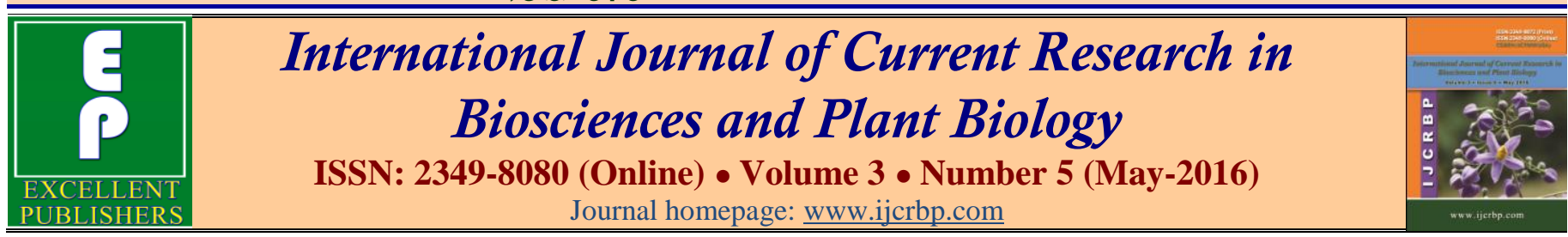

\title{
Identification of Pseudoknot in the Intergenic Region between VP30 and VP24 Gene of Ebola Virus and Designing Aptamer against the Pseudoknot
}

\author{
Damayanti Chakravarty', Pankaj K. Singh²*, Sabdar Rahaman³ and Sayak Ganguli4 \\ ${ }^{1}$ Department of Food Science and Nutrition Management, J.D. Birla Institute, Kolkata-70o oo2, India \\ ${ }^{2}$ Bioinformatics Division, The Biome, Kolkata-70o 064, India \\ 3 Department of Botany, Bangabasi Evening College, Kolkata-70o oog, India \\ 4Theoretical and Computational Biology Division, Amplicon Institute of Interdisciplinary Science and Technology, \\ Palta-743 122, India
}

*Corresponding author.

\begin{abstract}
A b s t r act
Ebola has been identified to be one of the most ominous pathogens capable of causing pandemic fatal disease in humans. Efforts are on to control the pathogen using various different therapeutic techniques and the vaccination effort is in its early second phase according to WHO sources. However, whether the candidate vaccine can confer "herd immunity" to a particular population remains a doubt. Thus alternative therapeutic approaches should be continuously explored, so as to combat the spread of the disease. In this work we attempt to identify one of the most predominant and essential RNA structural motifs - pseudoknot in the entire Ebola genome. The results indicate that a conserved pseudoknot motif is present in between the intergenic regions in between VP 24 and VP 30.
\end{abstract}

\section{Article Info}

Accepted: 12 April 2016

Available Online: 06 May 2016

Ke ywords

Antisense aptamer

Ebola virus

Pseudoknot

\section{Introduction}

Ebola virus, a member of Filoviridae virus family, is known as emerging and re-emerging zoonotic pathogen. It causes acute haemorrhagic fever with high case-fatality rate in humans (up to 90\%) reported by Marzi et al. (2011). It was first reported in Democratic Republic of the Congo (formerly Zaire) in 1976. The virus was named after the Ebola River where it was first discovered. Five strains of Ebola virus have been identified namely- Zaire Ebola virus (ZEBOV), Sudan Ebola virus (SEBOV), Côte d'Ivoire Ebola virus (CIEBOV), Reston Ebola virus (REBOV) and the Bundibugyo Ebola virus reported by Moghdam et al. (2015). The virion is filamentous, $970 \mathrm{~nm}$ long, $80 \mathrm{~nm}$ in diameter and enveloped. The genome is approximately 19 kilobase negative-sense single-stranded RNA. Ebola virus's genomes encode seven structural proteins (namely: NP, VP35, VP40, VP30, VP24 and L) and two non-structural soluble glycoproteins (GP): Soluble GP and Small Soluble GP. Base-pairing of a single-stranded region of RNA in the loop of a hairpin to a stretch of complementary nucleotides elsewhere in the RNA chain leads to formation of a Pseudoknot studied by Cliej et al. (1985).

Pseudoknots have roles in the replication cycles of numerous animal and plant viruses, including, in humans, the Flavivirus hepatitis C virus (HCV) (Wang et al., 1995), the Coronavirus which is responsible for Severe Acute Respiratory Syndrome (SARS-CoV) reported by Thiel et al. (2003), the oncogenic retrovirus T-cell lymphotrophic virus types I and II14, and certain strains of HIV reported by Brail et al. (2003). 
Pseudoknots act in the regulation of initiation of protein synthesis and in template recognition by the viral replicase. In coding regions they modulate the elongation and termination steps of translation. In the 5' NCRs, these motifs have roles in the regulation of translation initiation and on the other hand in the coding region they affect translation elongation. Pseudoknots have also been identified in the catalytic RNAs of some RNA satellite viruses, where they have a role in genome replication reported by Taylor et al. (2003).

At this juncture, the present study focuses on designing aptamers against specific genomic landmarks in the Ebola genome. Development of inhibitors that target key components in pathogens often requires detailed knowledge of structure and function or screening of large compound libraries with highly sensitive reporter(s). Nucleic acid aptamers provide an alternative method for inhibitor development as well as target validation. Aptamer identification and characterization does not require detailed knowledge of the target (Binning et al., 2012; Bunka et al., 2010; Lee et al., 2006; Okonechikov et al., 2012). However, in situations where detailed structural and functional information are available, aptamer development can be tailored to enhance the impact of aptamer binding by targeting aptamer interactions to functionally important regions.

Single-stranded DNA or RNA (ssDNA or ssRNA) molecules, which can bind to pre-selected targets including proteins and peptides with high affinity and specificity, are known as Aptamers. They possess versatility in binding to diverse targets, that is, they assume a variety of shapes due to their propensity to form helices and single-stranded loops. They are used as sensors and therapeutic tools. They are also used to regulate cellular processes, as well as to guide drugs to their specific cellular targets. Their specificity and characteristics are determined by their tertiary structure, not by their primary sequence. Over the years aptamer based therapeutics have evolved as we have gained understanding regarding the methods for avoiding nuclease sensitivity, poor bioavailability, fast renal clearance and limited cellular uptake.

\section{Materials and methods}

The complete genomes of all the Ebola virus strains were curated from NCBI Genome database. Genes and Intergenic regions from each virus's genome (3'UTR, 5'UTR, GP, L, NP, VP24, VP30, VP35, VP40,
Intergenic region between VP24 and L, Intergenic region between VP30 and VP 24, Intergenic region between VP40 and GP, Intergenic region between NP and VP35) were curated separately, aligned and consensus were generated using UGENE developed by Lee et al. (2006).

Dotknot Server designed by Sperschneider and Datta (2004) was used for identifying Pseudoknot in the consensus sequences. S Oligo server designed by Ding et al. (2004) was used for designing aptamer against the Pseudoknot. The Pseudoknot and the Aptamer was docked using the PatchDock Software designed by Dunhovny et al. (2002 and 2005).

Interaction between the two macromolecules was studied using DSSR: an Integrated Software Tool for Dissecting the Spatial Structure of RNA designed by Jing and Olson (2008). The macromolecular structures were visualised using the tool RasMol developed by Roger and Milne-White (1995).

\section{Results}

A Pseudoknot was identified in the Intergenic region between VP30 and VP24 (Position 109 - 127, Estimated Free Energy $=127 \mathrm{Kcal} / \mathrm{mol})($ Figs.1-3; Table 1). The aptamer designed using S Oligo was docked with the Pseudoknot.

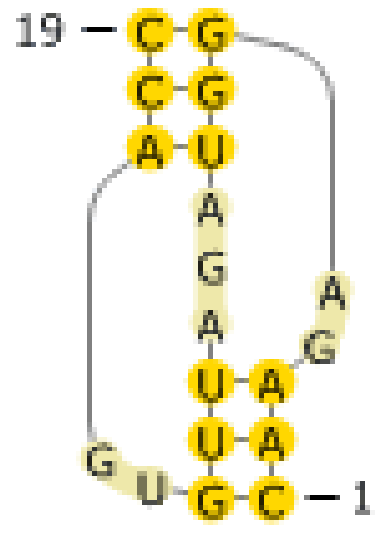

Fig. 1: Structure of Pseudoknot in Intergenic region between VP30 And VP24 visualised by using Pseudoviewer developed Han and Byun (2002 and 2003). 


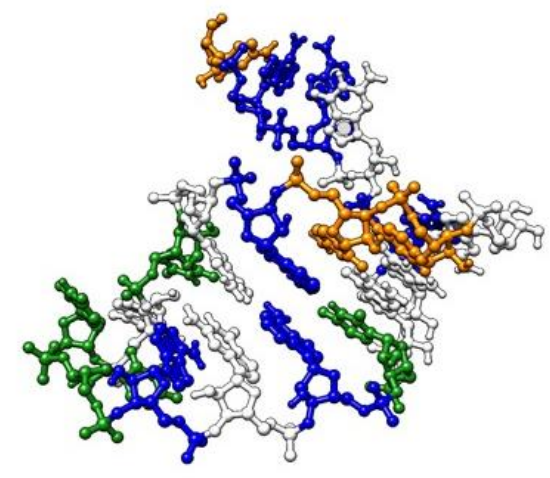

A

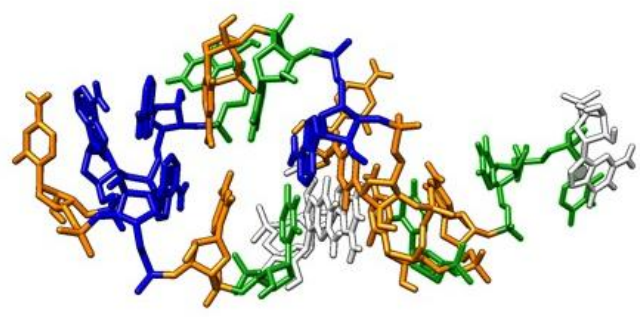

C

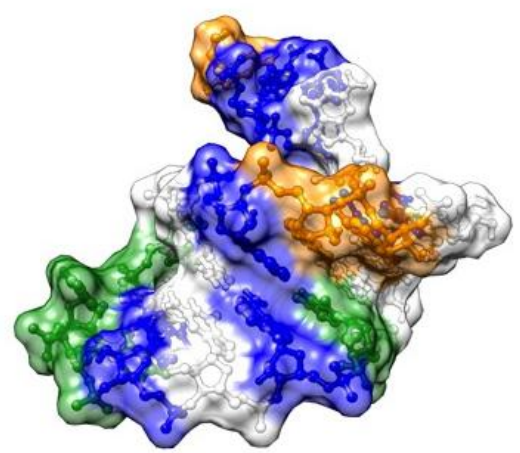

B



D

Fig. 2: Pseudoknot between VP 30 and VP 24 showing backbone and accessible surface area (A and B) and designed aptamer against the pseudoknot showing its backbone and accessible surface area (C and D). Adenine: blue, Cytosine: orange, Guanine: white and Uracil: green.
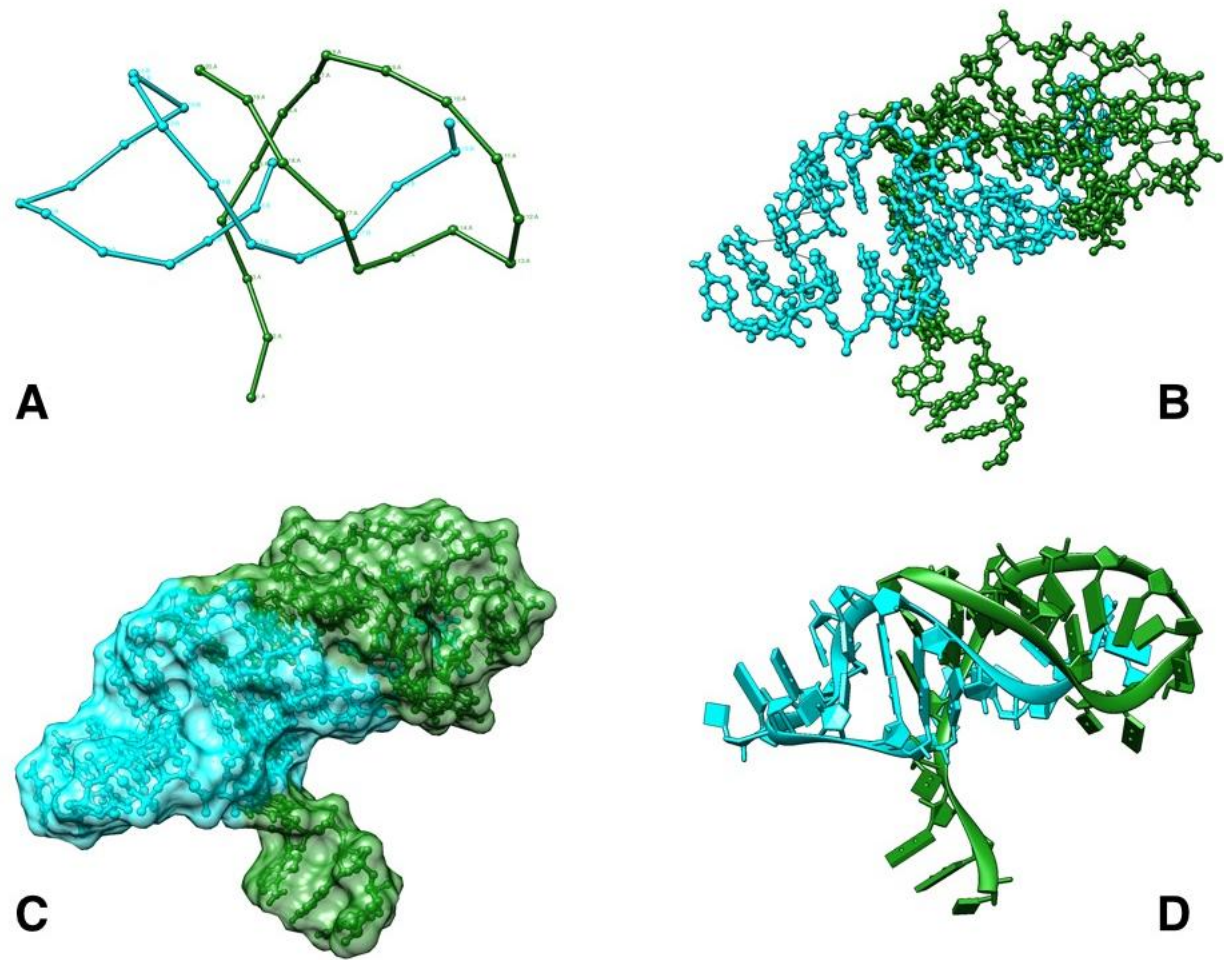

Fig. 3: Docking complex of pseudoknot (green) and aptamer (cyan). Backbone trace (A), Ball and Stick with hydrogen bonds in black (B), Ribbon showing conformation of interactions (C) and Surface Interactions (D). After analysing the docking result using DSSR, thirteen base pairings were found between Pseudoknot and Aptamer. 
Table 1. List of base pairings between pseudoknot and aptamer.

\begin{tabular}{ll}
\hline Pseudoknot & Aptamer \\
\hline A5 & U18 \\
G6 & C15 \\
G6, G20 & U16 \\
G7 & G3, C14 \\
A9 & U12 \\
G10 & C5 \\
G10 & A6 \\
G16 & C5 \\
A17 & U4 \\
C18 & G3 \\
C19 & G2 \\
\hline
\end{tabular}

\section{Discussion}

Over the years RNA peudoknots have been most studied and researched structural motifs in RNA biology. It is found in almost all classes of RNA and was first identified in Turmic Yellow Mosaic Virus, Plant viral genomes, as reported by Brierley et al. (2007). As a structure it is composed of several distinct topologies out of which the most characterized is the H-type where the bases of the hairpin loop in pair with external stem bases resulting in a second stem loop structure conformally forming the pseudoknot as indicated in Staple and Butcher (2005). Dey et al. (2011) have reported the presence of these ancient regulons in Human snRNPs. Chen et al. (2009) have reported the role of pseudoknot structures in translation initiation, elongation and frame shifting. Solinska (2014) investigated the therapeutic targeting of RNA structural motifs in RNA viral genomes and identified pseudoknots to be the most abundant RNA motifs. The identification of this pseudoknot structure in the Ebola viral genome is of significant importance as not only it sheds light on translational control and expression in Ebola but also emphasizes the regulatory function of such a common motif in the genome.

\section{Conclusion}

A Pseudoknot motif was identified and aptamer was designed against it. Significant interactions between the Aptamer and Pseudoknot was observed which indicate that the designed aptamer may serve as effective therapeutic leads for inhibiting the transcription, normal genome function as well as pathogen - host interactions. This should be clinically tested and subsequently evaluated for the same.

\section{Conflict of interest statement}

Authors declare that they have no conflict of interest.

\section{Acknowledgement}

Authors would like to express their gratitude towards The Biome management for providing laboratory space and infrastructure for completion of this work.

\section{References}

Baril, M., Dulude, D., Steinberg, S. V., BrakierGingras, L., 2003. The frameshift stimulatory signal of human immunodeficiency virus type 1 group $\mathrm{O}$ is a pseudoknot. J. Mol. Biol. 331, 571-583.

Binning, J.M., Leung, D.W., Amarasinghe, G.K., 2012. Aptamers in virology: recent advances and challenges. Front. Microbiol. 3, 29.

Brierley, I., Pennell, S., Gilbert, R.J., 2007. Viral RNA pseudoknots: versatile motifs in gene expression and replication. Nat. Rev. Microbiol. 5, 598-610.

Bunka, D.H., Platonova, O., Stockley, P.G., 2010. Development of aptamer therapeutics. Curr. Opin. Pharmacol. 10, 557-562.

Chen, G., Chang, K.Y., Chou, M.Y., Bustamante, C., Tinoco, I. Jr., 2009. Triplex structures in an RNA pseudoknot enhance mechanical stability and increase efficiency of -1 ribosomal frameshifting. Proc. Nat. Acad. Sci. (USA) 106, 12706-12711.

Dey, S. K., Ganguli, S., Roy, P., Basu, P., Chakraborty, H. J., Datta, A., 2011. Pseudoknots in human snRNPs. Int. J. Bioinf. Res. 3(1), 194-199.

Ding, Y., Chan, C.Y., Lawrence, C.E., 2004. S-fold web server for statistical folding and rational design of nucleic acids. Nucl. Acids Res. 32 (Web Server issue), W135W141. 
Duhovny, D., Nussinov, R., Wolfson, H.J., 2002. Efficient unbound docking of rigid molecules. In: Proceedings of the $2^{\text {nd }}$ Workshop on Algorithms in Bioinformatics (WABI) Rome, Italy (Eds.: Gusfield et al.). Lecture Notes in Computer Science 2452, Springer Verlag. pp. 185-200.

Han, K., Byun, Y., 2003. PseudoViewer2: visualization of RNA pseudoknots of any type. Nucl. Acids Res. 31(13), 3432-3440.

Han, K., Lee, Y., Kim, W., 2002. PseudoViewer: Automatic visualization of RNA pseudoknots. Bioinf. 18, S321-S328.

Kang, K.N., Lee, Y.S., 2012. RNA Aptamers: A review of recent trends and applications. Adv. Biochem. Eng. Biotechnol. 131, 153-169.

Lee, J.F., Stovall, G.M., Ellington, A.D., 2006. Aptamer therapeutics advance. Curr. Opin. Chem. Biol. 10, 282-289.

Marzi, A., Feldmann, H., Geisbert, T.W., Falzarano, D., 2011. Vesicular stomatitis virus-based vaccines for prophylaxis and treatment of filovirus infections. J. Bioterrior. Biodeg. 2011; doi; 10.4172/ 2157-2526.51-004

Moghadam, S.R.J., Omidi, N., Bayrami, S., Moghadam, S.J., Alinaghi, S.A.S., 2015. Ebola viral disease: a review literature. Asian Pac. J. Trop. Biomed. 5(4), 260-267.

Okonechnikov, K., Golosova, O., Fursov, M., The UGENE Team, 2012. Unipro UGENE: A unified bioinformatics toolkit. Bioinf. 28, 1166-1167.

Pleij, C. W., Rietveld, K., Bosch, L. A., 1985. New principle of RNA folding based on pseudoknotting. Nucl. Acids Res. 13, 1717-1731.
Roger, S., Milner-White, E.J., 1995. RasMol: Biomolecular graphics for all. Trends Biochem. Sci. (TIBS), 20(9), 374.

Schneidman-Duhovny, D., Inbar, Y., Nussinov, R., Wolfson, H.J., 2005. PatchDock and SymmDock: servers for rigid and symmetric docking. Nucl. Acids Res. 33, W363-367.

Solinska, J.S., 2014. Therapeutic targeting of structural RNA motifs in viral RNA genomes. Int. J. Virol. AIDS. 1, 001e.

Sperschneider, J., Datta, A., 2010. DotKnot: Pseudoknot prediction using the probability dot plot under a refined energy model. Nucl. Acids Res. 38(7), e103.

Staple, D.W., Butcher, S.E., 2005. Pseudoknots: RNA structures with diverse functions. PLoS Biol. 3, e213.

Taylor, J. M., 2006. Structure and replication of hepatitis delta virus RNA. Curr. Top. Microbiol. Immunol. 307, 1-23.

Thiel, V., Ivanov, K.A., Putics, A., Hertzig, T., Schelle, B., Bayer, S., Weissbrich, B., Snijder, E.J., Rabenau, H., Doerr, H.W., Gorbalenya, A.E., Ziebuhr, J., 2003. Mechanisms and enzymes involved in SARS coronavirus genome expression. J. Gen. Virol. 84, 2305-2315.

Wang, C., Le, S. Y., Ali, N., Siddiqui, A., 1995. An RNA pseudoknot is an essential structural element of the internal ribosome entry site located within the hepatitis $\mathrm{C}$ virus 5' noncoding region. RNA 1, 526-537.

Xiang, J.L., Olson, W.K., 2008. 3DNA: a versatile, integrated software system for the analysis, rebuilding and visualization of three-dimensional nucleic-acid structures. Nat. Protoc. 3(7), 1213-1227.

\section{How to cite this article:}

Chakravarty, D., Singh, P. K., Rahaman, S., Ganguli, S., 2016. Identification of pseudoknot in the intergenic region between VP30 and VP24 gene of Ebola virus and designing aptamer against the pseudoknot. Int. J. Curr. Res. Biosci. Plant Biol. 3(5), 54-58.

doi: http://dx.doi.org/10.20546/ijcrbp.2016.305.008 